\title{
ANÁLISE BIBLIOMÉTRICA DA REVISTA TECNOLOGIA E AMBIENTE (1995-2018)
}

\section{Altamir Rocha Antunes \\ Biólogo, Doutorando \\ PPGCA/UNESC. \\ E-mail: \\ altamirmad@hotmail.com \\ Aline Votri Guislon \\ Bióloga, Doutoranda PPGCA/UNESC. \\ E-mail:vg_aline@hotmail.com}

Amanda Vieira Matiola

Bióloga, Mestranda PPGCA/UNESC.

E-mail:

amanda.matiola1@gmail.com

Bruna Baldessar Ghislandi Bióloga

E-mail:

bruna_bghislandi@hotmail.com

\section{Iara Zaccaron Zanoni \\ Bióloga, Mestranda PPGCA/UNESC. \\ E-mail: \\ iara_zanoni@hotmail.com}

Nilzo Ivo Ladwig

Professor do Programa de

Pós-Graduação em Ciências

Ambientais - UNESC. E-mail: ladwig@unesc.net

Robson dos Santos

Professor do Programa de

Pós-Graduação em Ciências

Ambientais - UNESC. E-mail: robson@unesc.net

\section{Guilherme Alves Elias}

Professor Colaborador do Programa de

Pós-Graduação em Ciências Ambientais - UNESC

E-mail: guilherme@unesc.net

\section{BIBLIOMETRIC ANALYSIS OF THE JOURNAL TECNOLOGIA E AMBIENTE (1995-2018)}

\section{Resumo}

O desenvolvimento de estudos que reúnem informações sobre uma determinada área vem sendo do interesse de pesquisadores. Estes estudos trazem dados que mostram a evolução de temas de várias áreas, auxiliando no planejamento de novas pesquisas. O objetivo deste estudo foi caracterizar a produção científica da revista Tecnologia e Ambiente, desde sua criação, em 1995, até 2018, por meio de uma análise bibliométrica. A análise trouxe um panorama sobre as publicações da revista Tecnologia e Ambiente. Foram levantadas informações das áreas de pesquisa, autores mais influentes, publicações por ano, Universidades com maior número de publicações e palavras-chave mais utilizadas. Estes resultados confirmaram a importância da revista em nível regional, e autores e Universidades mais relevantes no processo de consolidação da revista. Ainda, foi possível obter uma visão sobre a produção científica e qual o perfil da produtividade científica desenvolvida até o momento.

Palavras-Chave: Arqueologia; Cienciometria; Meio ambiente; Mineração de carvão; Produção científica; Redes de colaboração; Santa Catarina.

\section{Abstract}

The development of studies that unite information from a given area has been of interest to researchers. These studies bring data that show the evolution of themes from various areas, supporting the planning and progress of new research. This paper aimed to carry out a bibliometric analysis of journal Tecnologia e Ambiente, from its creation in 1995 until 2018. Our analysis provided an overview of the publications of the journal Tecnologia e Ambiente. Information was collected from research areas, most influential authors, publications per year, Universities with the highest number of publications and most used keywords. Our results confirmed the importance of the journal at regional level, and the authors and collabo-rating universities most relevant in the process of consolidation of the journal. In addition, it was possible to obtain an insight into scientific production and the profile of scientific productivity developed up to now.

Keywords: Archeology; Coal mining; Cooperation networks; Environment; Santa Catarina; Scientific production; Scientometrics. 


\section{INTRODUÇÃO}

O desenvolvimento dos estudos bibliométricos está relacionado com a evolução da Ciência da Informação e contribui diretamente para a avaliação da produção do conhecimento científico nas diversas áreas do conhecimento (MONGE-NÁJERA; HO, 2016; ALVAREZ; CAREGNATO, 2017). Atualmente, os periódicos científicos são os principais meios de divulgação do conhecimento, publicando novas descobertas, avanços e novas abordagens teóricas, permitindo a disseminação de informações confiáveis (ANNA, 2018).

Os estudos bibliométricos sobre periódicos podem fornecer informações relevantes sobre a evolução nas publicações auxiliando no planejamento e progresso de uma revista (MACHADO et al., 2015; MONGE-NÁJERA; HO, 2016; ZHENG et al., 2017). Uma vez que, quando os periódicos são regionalmente especializados, se tornam responsáveis por divulgar o progresso científico de determinada região geográfica.

Neste contexto, a revista Tecnologia e Ambiente ocupa um lugar de destaque no âmbito do estado de Santa Catarina, bem como no sul do Brasil. O periódico, que está vinculado a Universidade do Extremo Sul Catarinense (UNESC), uma instituição comunitária localizada no extremo sul do Estado, publica artigos voltados a área interdisciplinar com ênfase em meio ambiente e desenvolvimento; sociedade e humanidades; engenharia, tecnologia e gestão; ciências agrárias, biológicas e da saúde (TECNOLOGIA E AMBIENTE, 2019).

A região sul de Santa Catarina é conhecida por ser uma região carbonífera, devido à intensa explotação de carvão mineral ocorrida desde o século passado (BELOLLI; QUADROS; GUIDI, 2002). Desde então, a região tem seu histórico ambiental marcado por extensas áreas degradadas, além de mudanças drásticas na paisagem (LADWIG; DAGOSTIM; BACK, 2018). Com isso, pesquisadores de diversas áreas e instituições vêm desenvolvendo estudos voltados à problemática ambiental da região (BORTOLOTTO; SAVI; PICH, 2007; ROSADO et al., 2014; PEZENTE; SANT'ANA, 2018).

A revista Tecnologia e Ambiente é precursora da temática sobre o meio ambiente na região, onde seu primeiro volume, lançado em 1995, contém trabalhos voltados a discutir e analisar o cenário energético internacional da época (QUEIROZ, 1995) e a preocupação com a questão hídrica (ALEXANDRE; KREBS; VIERO, 1995; 
ALEXANDRE et al., 1995) com a melhoria da qualidade ambiental da região (BORTOT; ALEXANDRE, 1995).

No período de 1995 a 2006 a revista teve suas publicações em formato impresso e, a partir de 2007, passou a publicar também em formato eletrônico, completando, em 2018, seu $24^{\circ}$ volume. O objetivo deste estudo foi caracterizar a produção científica da revista Tecnologia e Ambiente, desde sua criação, em 1995, até 2018, por meio de uma análise bibliométrica. A partir disso, espera-se compreender as tendências e padrões das publicações da revista, fazendo um paralelo com a situação científica histórica e atual da região sul do Estado de Santa Catarina.

\section{MATERIAL E MÉTODOS}

Foram considerados todos os artigos e notas técnicas publicados na revista Tecnologia e Ambiente (ISSN impresso 1413-8131 e eletrônico 2358-9426), desde 1995 a 2018, tanto em meio impresso quanto eletrônico. Os indicadores bibliométricos analisados foram: (i) ano de publicação, (ii) palavras-chave, (iii) autor, (iv) gênero dos autores, (v) instituição, (vi) categoria e (vii) idioma do manuscrito.

Todos os trabalhos foram categorizados após leitura do resumo, sendo que as categorias foram previamente estabelecidas de acordo com quatro grandes áreas do conhecimento: Engenharias (incluindo trabalhos de tecnologias e ciências agrárias, com foco principal em engenharia); Ciências Biológicas (trabalhos voltados à biologia de plantas e animais, ecologia, plantas medicinais e recuperação de áreas degradadas); Ciências da Saúde (trabalhos desenvolvidos no âmbito da saúde em geral) e Ciências Sociais (trabalhos com temas voltados a sociedades e humanidades).

Para obtenção dos dados referentes aos trabalhos publicados até o ano de 2006 recorreu-se ao acervo físico da revista, localizada na Biblioteca Central da UNESC. Os trabalhos publicados a partir de 2007 foram consultados diretamente no acervo digital da revista. No total de 24 volumes publicados, 12 foram analisados em material impresso e 12 em meio eletrônico. Para a instituição, foi considerada a frequência de cada uma quando pelo menos um manuscrito possuía um membro filiado. Para análise e apresentação da rede de colaboração das instituições utilizou-se o programa Gephi 0.9.2, com base no algoritmo de Fruchterman e Reingold (1991). 
Para a criação do Cluster Words, utilizou-se o programa R (R CORE TEAM, 2018), pacote igraph/network, utilizando-se as palavras-chave indicadas em cada manuscrito.

\section{RESULTADOS E DISCUSSÃO}

Foram registrados 239 trabalhos científicos desde 1995 até o ano de 2018. Desses trabalhos participaram no total 414 autores, sendo 175 (40\%) mulheres e 239 (60\%) homens, o que indica pouca disparidade de gênero nos artigos da revista Tecnologia e Ambiente. Padrão semelhante foi encontrado na revista Latinoamericana de Investigación en Matemática Educativa (RELIME), onde entre os autores 51\% são homens e $49 \%$ mulheres (MACHADO et al., 2015).

Estudos mostram que a tendência ao equilíbrio de gênero nas pesquisas científicas é crescente, mas existem diferenças nas áreas do conhecimento em que atuam, sendo que os homens predominam nos setores de engenharias e tecnologias aplicadas e as mulheres predominam nos setores de humanidades, sociais e saúde (MELO; LASTRES; MARQUES, 2004; GARCIA; DUARTE, 2017). Entretanto, o presente estudo mostrou que o segundo e terceiro lugares no ranking de publicações da revista Tecnologia e Ambiente são ocupados por mulheres e que publicam nas áreas de Engenharias e Ciências Biológicas.

A distribuição anual de trabalhos publicados na revista revelou que ao longo dos seus 24 anos de atividade, houve flutuações no número de publicações (Figura 1). É comum a tendência ao crescimento no número de publicações nas revistas científicas e as flutuações também são presentes e podem variar em relação ao ano (MONGE-NÁJERA; HO, 2016; ZHENG et al., 2017; BOAMAH; HO, 2017; ABDI et al., 2018), mostrando períodos de mudanças ou adaptações dos periódicos.

Neste caso, houve um decréscimo no número de trabalhos publicados no período de 2004 a 2012, e um rápido aumento de trabalhos publicados a partir de 2013. As flutuações ocorreram pelas adaptações da revista, visto que até o ano de 2013 as publicações eram disponibilizadas em dois números anuais, essa transição resultou em alguns volumes com número inferior de publicações anuais. Só a partir do ano de 2012 a revista reestabeleceu um número regular de publicações. 
Figura 1 - Distribuição anual do número de trabalhos publicados na revista Tecnologia e Ambiente.

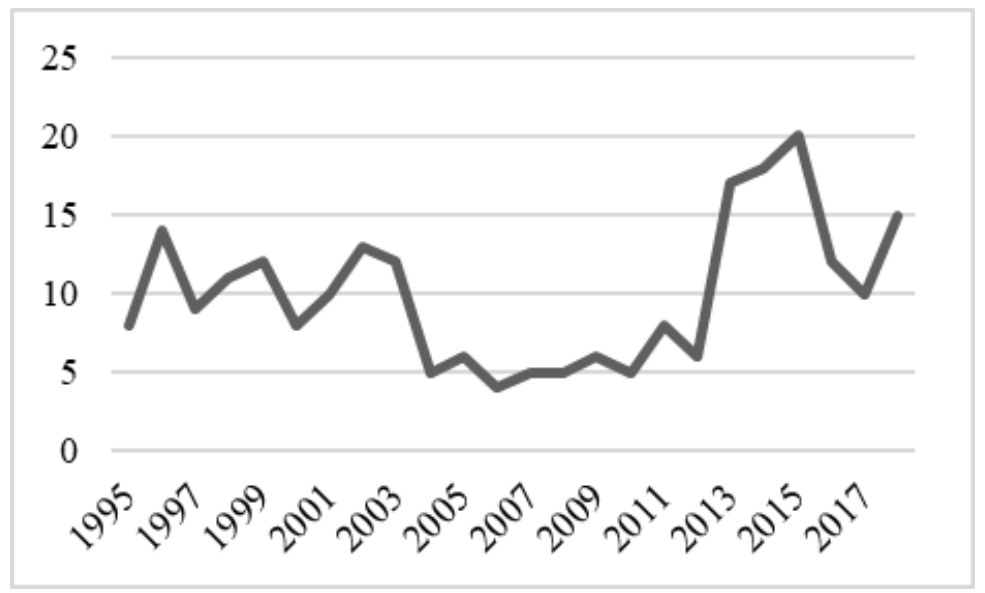

Fonte: Elaborado pelos autores.

A maioria dos autores participou de apenas um artigo equivalendo a 84,1\% do total analisado. Os autores envolvidos com número maior que sete artigos, representaram 0,9\% (Tabela 1). Os resultados corroboram com a lei de Lotka (1926), em que se observa uma concentração de publicações em um grupo específico e uma difusão entre os autores, sendo imperceptível revelar quais seriam os mais relevantes apenas analisando a quantidade de publicações. Esse resultado, de acordo com o padrão de Lotka, mostra que existe um número baixo de autores especialistas em cada área.

Tabela 1 - Número de publicações por autores em comparação ao padrão de Lotka

\begin{tabular}{cccc}
\hline $\mathrm{N}^{\mathbf{o}}$ de artigos por autor & Frequência & Representatividade & Padrão Lotka \\
\hline 1 & 367 & 84,1 & 61,0 \\
2 & 48 & 11,0 & 15,2 \\
3 & 11 & 2,5 & 6,8 \\
4 & 2 & 0,5 & 3,8 \\
5 & 2 & 0,5 & 2,4 \\
6 & 2 & 0,5 & 1,7 \\
7 & 0 & 0,0 & 1,2 \\
Mais de 7 & 4 & 0,9 & 8,0 \\
\hline Total & - & $100 \%$ & $100 \%$
\end{tabular}

Fonte: Elaborado pelos autores. 
Entre os pesquisadores com o maior número de publicações, destacam-se os autores da área de Engenharias e Ciências Biológicas (Tabela 2), classificadas nesse estudo (Figura 2).

Tabela 2 - Lista dos 10 autores com maior número de publicações na revista Tecnologia e Ambiente, seguidos das suas respectivas áreas de concentração.

\begin{tabular}{ccc}
\hline Autores & $\mathrm{N}^{\mathrm{o}}$ de artigos & Área de Concentração \\
\hline Back, A J & 21 & Engenharias \\
Alexandre, N Z & 12 & Engenharias \\
Citadini-Zanette, V & 12 & Ciências Biológicas \\
Zocche, J J & 9 & Ciências Biológicas \\
Campos, J B & 6 & Ciências Sociais \\
Ladwig, N I & 6 & Engenharias \\
Gonçalves, T M & 6 & Ciências Sociais \\
Santos, R & 5 & Ciências Biológicas \\
Dufloth, J H & 4 & Engenharias \\
Queiroz, G C & 4 & Engenharias \\
\hline
\end{tabular}

Fonte: Elaborado pelos autores.

Figura 2 - Classificação de áreas dos artigos publicados na revista Tecnologia e Ambiente entre o período de 1995-2018.

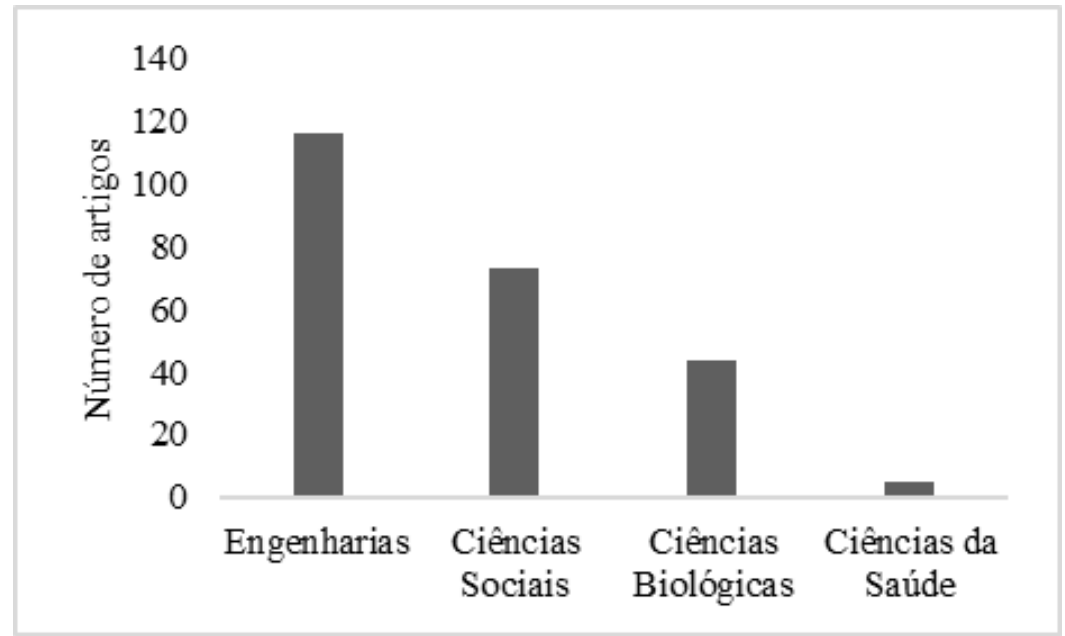

Fonte: Elaborado pelos autores.

Com um total de 21 artigos, o equivalente a 9\% das publicações, Back, A J é o autor com maior produção na revista. Graduado em Agronomia, atua em pesquisas sobre temas como hidrologia, recursos hídricos e agrometeorologia. Além de professor da UNESC, também é pesquisador da Empresa de Pesquisa Agropecuária e Extensão Rural de Santa Catarina (EPAGRI), ocasionando em forte ocorrência destas instituições na rede de colaborações (Figura 3). 
Ocupando o segundo lugar entre os autores com maior publicação, Alexandre, $\mathrm{N}$ Z publicou seus artigos na revista Tecnologia e Ambiente no período de 1995 a 2002, enquanto atuava como professora e pesquisadora da UNESC. Graduada em Química, sua atuação em pesquisas voltava-se às áreas de química ambiental e controle de poluição. Também em segundo lugar no número de publicações, a autora Citadini-Zanette, V, graduada em Ciências Biológicas e vinculada a UNESC como professora, contribuiu com suas pesquisas sobre a vegetação regional, com viés para diagnósticos ambientais, recuperação de áreas degradadas e etnobotânica de espécies vegetais da região.

O terceiro autor no ranking dos artigos é Zocche, J J, também graduado em Ciências Biológicas e professor da UNESC, contribuiu para a revista com estudos de vegetação e fauna da região, além de participar também em pesquisas sobre recuperação de áreas degradadas pela mineração de carvão e arqueologia.

Figura 3 - Rede de colaboração entre as Instituições que publicaram na revista Tecnologia e Ambiente entre o período de 1995-2018.

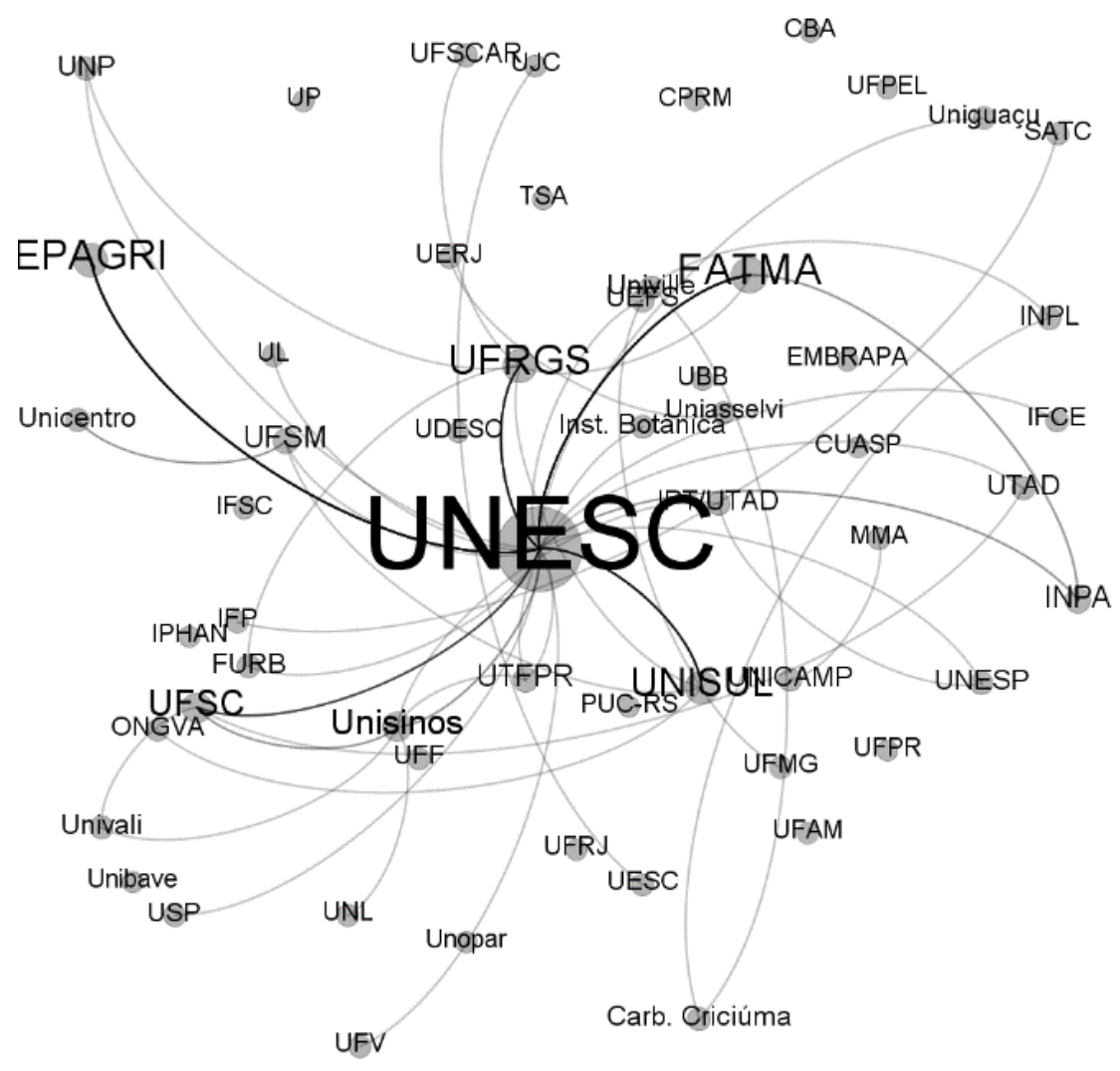

Fonte: Elaborado pelos autores. 
Estes foram os principais pesquisadores envolvidos nas publicações e que alavancaram a produção e o progresso da revista Tecnologia e Ambiente. Salienta-se que entre os oito autores com maior produção na revista, todos possuíam vínculo com a UNESC. Essas publicações são em maioria resultados de projetos voltados principalmente ao contexto ambiental do sul do Brasil, contribuindo, desta forma, para a tomada de decisões e desenvolvimento regional.

Dentre os 239 artigos publicados até 2018 em cada área do conhecimento (Figura 2), 116 artigos são referentes a grande área de Engenharia, com destaque para temas de engenharia ambiental, agronomia, resíduos industriais e geoprocessamento. A área das Ciências Sociais ocupou o segundo lugar com 73 artigos, relacionados a temas diversos, como discursos sobre sustentabilidade e empresas, destacando os temas sobre arqueologia e patrimônio histórico e cultural, que tiveram forte ascensão na revista desde 2011.

A área das Ciências Biológicas ocupou o terceiro lugar com 44 publicações, estas voltadas principalmente a ecossistemas naturais e seus componentes de fauna e vegetação, mas também no contexto da recuperação ambiental. A área das Ciências da Saúde apresentou cinco artigos, voltados a questões de saúde pública, sendo dois deles relacionados à mineração de carvão. Isso mostra que a temática do carvão desperta interesse dos pesquisadores e instituições que publicam na revista Tecnologia e Ambiente, permeando todas as áreas do conhecimento tratadas no periódico.

A distribuição das áreas de estudos revelou a tendência de investigação da revista Tecnologia e Ambiente, onde a área de Engenharia, mesmo com um número total de trabalhos superior em relação as demais áreas, vem perdendo força para a área de Ciências Sociais, que se mostrou crescente nas publicações a partir de 2011 (Figura 4). O avanço das Ciências Sociais a partir de 2011 coincide com o início das publicações do autor Campos JB, especialista na área de arqueologia, tema que vem crescendo nas pesquisas publicadas na revista, trazendo informações a respeito dos sítios arqueológicos presentes na região Sul de Santa Catarina (CEZARO et al., 2011; PAVEI et al., 2015). 
Figura 4 - Distribuição anual do número de trabalhos por área de concentração, publicados na revista Tecnologia e Ambiente entre o período de 1995-2018.

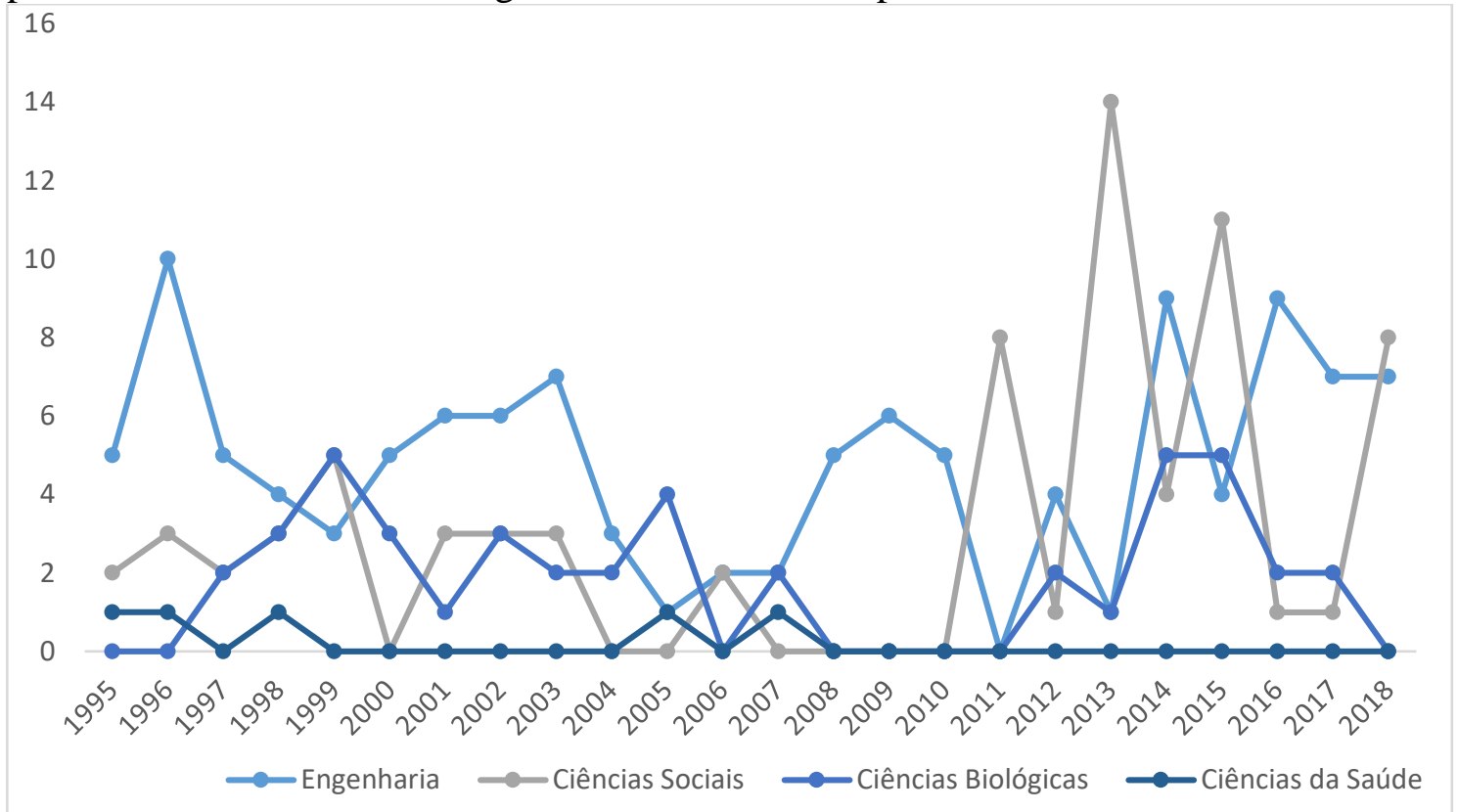

Fonte: Elaborado pelos autores.

Percebe-se que as áreas de concentração seguem a mesma tendência de publicação ao longo dos anos, embora em números de artigos a Engenharia sobreponha às demais (Figura 4). Destaque também para a área das Ciências da Saúde que teve um baixo número de publicações, sendo sua última publicação no ano de 2007, que, embora Bortolotto et al. (2007) tenham trazido a toxicidade in vitro, o tema tratava sobre a toxicidade mediante à exposição a água do carvão remetendo também aos estudos ambientais. Outra área que se destaca é a de Ciências Biológicas que a partir dos anos de 2011 aumentou consideravelmente as contribuições na revista.

Esse destaque foi uma consequência do aumento de artigos sobre fauna e vegetação em geral (CAZNOK; SANTOS; CITADINI-ZANETTE, 2005; SANTOS et al., 2006; MAFFIOLETTI et al., 2012; CITADINI-ZANETTE, 2014; SILVA, 2014; ROSADO et al., 2014; PATRÍCIO et al., 2014; PAVEI et al., 2015; VINHOLES et al., 2015; PADILHA et al., 2016; BOLLA et al., 2017; ESPILLERE; PEREIRA, 2017). Os artigos voltados as Ciências Sociais também tiveram aumento de publicações a partir do ano de 2006, abordando os impactos socioambientais nos municípios de Criciúma e Içara (VIRTUOSO; MILIOLI, 2006), valorização de patrimônio e educação patrimonial (SOARES; DIAS 2011; MATIAS et al., 2011; SCHWENGBER, 2011), impactos da Igreja Católica sobre colônias espanholas, abordando a arqueologia histórica (NIMMO, 
2013), a sustentabilidade sobre a inclusão de catadores de lixo em programas de gerenciamento integrados (GUADAGNIN et al., 2014), reflexões do patrimônio cultural (MACHADO, 2015) e indicadores de sustentabilidade (CAMARGO et al., 2018; MELO et al., 2018; WITT et al,. 2018).

Dentre as Instituições mais representativas em relação ao número de publicações (Tabela 3), a UNESC se destaca como principal instituição no número de artigos publicados, devido aos pesquisadores vinculados, que também correspondem aos autores com maiores publicações na revista. Cabe ressaltar que houve o registro de Universidades estrangeiras, como a Universidade de Londres e a Universidade de Trás-os-Montes e Alto Douro, resultado do vínculo entre os pesquisadores da UNESC e as Universidades estrangeiras.

Tabela 3 - Instituições responsáveis pelos maiores números de artigos publicados na revista Tecnologia e Ambiente entre o período de 1995-2018.

\begin{tabular}{llc}
\hline Instituição & Sigla & FR $\geq 6$ \\
\hline Univ do Extremo Sul Catarinense & UNESC & 146 \\
Univ Federal de Santa Catarina & UFSC & 24 \\
Emp de Pesq Agro e Ext Rur de Santa Catarina & EPAGRI & 17 \\
Fundação do Meio Ambiente & FATMA & 14 \\
Univ do Sul de Santa Catarina & UNISUL & 13 \\
Univ Federal de São Carlos & UFSCar & 10 \\
Univ Federal do Rio Grande do Sul & UFRGS & 9 \\
Univ Federal do Paraná & UFPR & 6 \\
Univ do Vale do Rio dos Sinos & UNISINOS & 6 \\
Univ de São Paulo & USP & 6 \\
\hline
\end{tabular}

Fonte: Elaborado pelos autores.

Pode-se observar a relação entre as instituições na revista Tecnologia e Ambiente. A rede da associação entre as universidades/instituições possui 24 nós com baixa conexão entre os componentes e 57 nós com alta conexão representados, principalmente, pelas relações entre UNESC, EPAGRI, FATMA e UNISUL, destaque para UNESC que se apresenta como o maior polo de publicações (Figura 4).

A UFSC, embora tenha uma alta frequência de artigos no periódico, poucos fazem conexões com outras universidades. Em contrapartida, Universidades com baixa frequência de artigos podem estabelecer conexões mais fortes, como a UNISINOS, UTFPR e INPA. A quantidade de conexões é um reflexo direto das publicações de artigos 
sobre a temática ambiental. As Universidades mais produtivas se destacam como as mais basais na rede de colaboração. Ainda, percebe-se uma tendência na área de Engenharia, onde o vínculo entre UNESC e FATMA são representados, principalmente, pela temática ambiental, caso da influência de mineração e a qualidade das águas superficiais (ALEXANDRE, 1996), das fontes de poluição (ALEXANDRE et al., 1996), diagnósticos ambientais (ALEXANDRE, 1999) e contaminação de recursos hídricos (ALEXANDRE, 2002). Em relação às publicações entre UNESC e Epagri, destacam-se estudos sobre modelagem de precipitações no estado de Santa Catarina (BACK, 1998), caracterização física de bacias hidrográficas (BACK; NEUBERT, 2000), estimativas de evapotranspiração (BACK, 2006), déficit hídrico nas regiões do Sul de Santa Catarina (DUFLOTH; BACK, 2012), e eficiência de irrigação em sistemas de cultivo coletivo (BACK; JUST, 2018).

Já para as relações entre UNESC e UNISUL os trabalhos estiveram, geralmente, relacionados a questões sociais, como as crises socioambientais (MILIOLI, 1995), outros estudos abordaram a cadeia produtiva têxtil (GOULARTI FILHO, 1996) e a diversidade florística e vegetação ciliar no Sul de Santa Catarina (MARCOMIN; CITADINIZANETTE, 1999). As publicações em colaboração da UNESC e UFRGS abordam todas as temáticas, como os estudos de tratamento de efluentes na mineração de carvão (ALEXANDRE et al., 1995), análises de riscos geológicos para a cidade de Porto Alegre (OLIVEIRA, 1996) e levantamento florístico de restinga arbórea (CITADINI-ZANETTE et al., 2001), resultado do vínculo entre os laboratórios de botânica entre as duas universidades.

Em relação às palavras-chave, verificou-se que: Santa Catarina, arqueologia, ambiental, água, biodiversidade, geodesia, gestão e análise, entre outras, foram as mais representativas (Figura 5) e possuem conotações semelhantes com o escopo da revista Tecnologia e Ambiente. A palavra-chave Santa Catarina apareceu em oito artigos e ocorreu por conta das publicações regionais, sobretudo do Sul do Estado. Arqueologia e meio ambiente apareceram sete vezes, esses resultados estão de acordo com as áreas de estudo e representam a temática de Ciências Sociais e Engenharias. Esta análise corrobora com os principais resultados relacionados às temáticas propostas neste estudo. 
Figura 5 - Word Cloud de palavras-chave dos artigos da revista Tecnologia e Ambiente

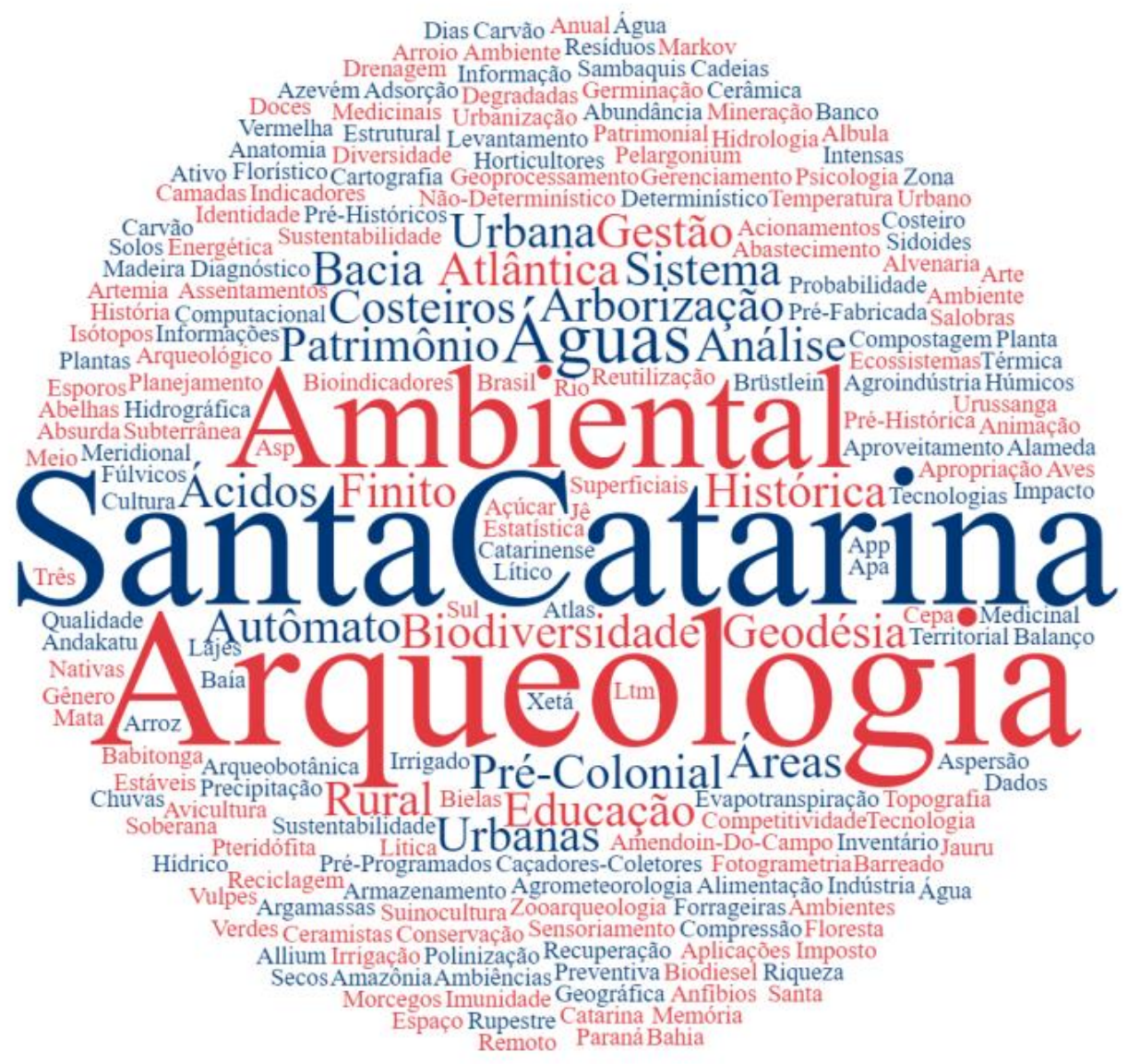

Fonte: Elaborado pelos autores.

Os artigos publicados na revista Tecnologia e Ambiente refletem muito dos temas de pesquisa trabalhados no Programa de Pós-Graduação em Ciências Ambientais (PPGCA) da UNESC, que reúne professores envolvidos em muitas pesquisas sobre a temática ambiental da região, representando também muitos dos autores e comitê editorial da revista. Neste contexto, Brofman (2012) salienta que uma revista científica local deve criar condições para garantir a qualidade e competitividade do periódico, além de outros critérios que valorizem a pesquisa em todas as suas dimensões e complexidades, comprometendo a instituição e seus integrantes com o aperfeiçoamento de suas pesquisas. Ainda nesse contexto da tomada de decisões para incentivar a melhoria da produção de um periódico científico, López (2017) recomenda que deva haver políticas editoriais que impeçam os periódicos de perder seu caráter científico e se tornem canais institucionais de comunicação. 
Revista Tecnologia e Ambiente, v. 27, 2021, Criciúma, Santa Catarina/SC - ISSN Eletrônico 2358-9426 e ISSN Impresso 1413-8131

\section{CONCLUSÃO}

Foi observada uma tendência no aumento de publicações na área das Engenharias, Ciências Sociais e Ciências Biológicas e uma diminuição nas publicações na área das Ciências da Saúde, resultando no fim das publicações nesta área no ano de 2007, provavelmente por conta de o escopo da revista ter modificado consideravelmente. Também foi observado que poucos pesquisadores são especialistas nas grandes áreas propostas nesse estudo, padrão confirmado por meio da comparação com o padrão de Lotka. Ao mesmo tempo foi possível registrar os autores mais influentes e com os maiores números de publicação. Outra questão importante abordada nesse estudo foi as redes de colaboração, onde foi possível observar que uma maior frequência de artigos no periódico não indica uma maior conexão entre instituições; ao passo que aquelas que apresentam baixa frequência de artigos podem estabelecer conexões mais fortes. Ainda sobre as conexões, foi possível atestar ligações entre UNESC, UFSC, UFRGS, EPAGRI e FATMA (Atualmente, Instituto do Meio Ambiente de Santa Catarina - IMA), que foram cruciais no processo de consolidação da revista Tecnologia e Ambiente.

\section{REFERÊNCIAS}

ABDI, A.; IDRIS, N.; ALGULIYEV, R.; ALIGULIYEV. Bibliometric Analysis of IP\&M Journal (1980-2015). Journal of Scientometric Research, v. 7, n. 1, p. 54-62, 2018.

ALEXANDRE, N. Z. Principais impactos ambientais gerados na indústria de revestimentos cerâmicos. Tecnologia e Ambiente, v. 2, n. 1, p. 73-84, 1996.

ALEXANDRE, N. Z. Diagnóstico ambiental da Região Carbonífera de SC: degradação dos recursos naturais. Tecnologia e Ambiente, v. 5, n. 2, p. 35-50, 1999.

ALEXANDRE, N. Z. Proposta de metodologia simplificada para investigar a contaminação dos recursos hídricos pela mineração de carvão: estudo de caso, sub-bacia do Rio Mãe Luzia. Tecnologia e Ambiente, v. 8, n. 1 p. 49-61, 2002.

ALEXANDRE, N. Z.; NOSSE, E. O.; MARTINELlO, C.; NETO, R. R.; NETO, A. C. B. Estudo de tratabilidade de efluentes líquidos de mineração de carvão com a utilização de materiais alternativos. Tecnologia e Ambiente, v. 1, n. 1, p. 75-90, 1995.

ALEXANDRE, N. Z.; KREBS, A. S. J.; VIERO, A. C. Qualidade das águas superficiais do município de Criciúma, SC - Dados preliminares. Tecnologia e Ambiente, v. 1, n. 2 , p. 13-18, 1995.

ALEXANDRE, N. Z.; KREBS, A. S. J.; VIERO, A. C. Metodologia aplicada para mapeamento de fontes de poluição - a experiência do município de Criciúma. Tecnologia e Ambiente, v. 2, n. 1, p. 63-72, 1996. 
ALVAREZ, G. R.; CARAGNATO, S. E. A Ciência da Informação e sua Contribuição para a avaliação do conhecimento científico. Revista do Instituto de Ciências Humanas e da Informação, v. 31, n. 1, p. 9-26, 2017.

ANNA, J. S. Mapeamento Sistemático na Base de Dados em Ciência da Informação: Periódicos Científicos em Discussão. Brazilian Journal of Information Science: research trends, v. 12, n. 1, p. 68-79, 2017.

BACK, A. J. Viabilidade da cobrança pela utilização de águas para irrigação na bacia do rio Araranguá. Tecnologia e Ambiente, v. 4, n. 2, p. 69-76, 1998.

BACK, A. J.; NEUBERT, E. O. Bacia Hidrográfica do Rio da Laje. Tecnologia e Ambiente, v. 6, n. 1, p. 15-24, 2000.

BACK, A. J. Coeficiente da equação de Makkink para estimativa da evapotranspiração de referência na região de Urussanga, SC. Tecnologia e Ambiente, v. 13, n. 1, p. 1-7, 2000 .

BACK, A. J.; JUST, M. C. Consumo de Água em Lavouras de Arroz Irrigadas em Sistema Coletivo. Tecnologia e Ambiente, v. 24, p. 133-145, 2018.

BELOLli, M.; QUADROS, J.; GUIDI, A. História do Carvão de Santa Catarina: 1790-1950. Criciúma: IOESC, 2002.

BOAMAH, P. O.; HO, Y. S. Bibliometric Analysis of Ghana Publications in the Science Citation Index Expanded. Revista de Biología Tropical, v. 66, n. 1, p. 106-121, 2017.

BÔLLA, D. A.; CERON, C.; CARVALHO, F.; DE MATIA, D. L.; LUIZ, M. R.; PANATTA, K. A.; PAVEI, D. D.; MENDONÇA, R. Á.; ZOCCHE, J. J. Mastofauna terrestre do Sul de Santa Catarina: mamíferos de médio e grande porte e voadores. Tecnologia e Ambiente, v. 23, p. 61-78, 2017.

BORTOT, A.; ALEXANDRE, N. Z. Programa de proteção e melhoria da qualidade ambiental da Bacia do Rio Tubarão e Complexo Lagunar. Tecnologia e Ambiente, v. 1, n. 1, p. 55-74, 1995.

BORTOLOTTO, T.; SAVI, G. D.; CLAUS T. P. Influência da mineração de carvão na toxicidade das águas superficiais do Rio Molha, Urussanga, SC. Tecnologia e Ambiente, v. 13, n. 1 p. 1-16, 2007.

BROFMAN, P. R. A Importância das Publicações Científicas. Cogitare Enfermagem, v. 17, n. 3, p. 419-421, 2012.

CAMARGO, O. F.; ROSSO, P. M.; LADWIG, N. I. Modais alternativos de passageiros. Tecnologia e Ambiente, v. 24, p. 181-196, 2018.

CAZNOK, J.; SANTOS, R.; CITADINI-ZANETTE, V. Árvores e arbustos das praças do município de Criciúma, Santa Catarina. Tecnologia e Ambiente, v. 11, n. 1, p. 101-114, 2005.

CEZARO, H. S.; BRAGA, A. S.; SANTOS, M. C. P.; ZOCCHE, J. J.; CAMPOS, J. B. A arte rupestre do extremo sul catarinense: o caso do Sítio Malacara I, Santa Catarina, Brasil. Tecnologia e Ambiente, v. 17, p. 133-149, 2011.

CITADINI-ZANETTE, V.; SANTOS, R.; SOBRAL, M. Levantamento florístico da vegetação arbustivo-arbórea em área ecotonal entre Restinga e Floresta Ombrófila Densa 
das Terras Baixas, Praia de Palmas, Governador Celso Ramos, Santa Catarina, Brasil. Tecnologia e Ambiente, v. 7, n. 1, p. 105-120, 2001.

CITADINI-ZANETTE, V.; SANTOS; R.; EMERICH, K. H.; PASETTO, M. R.; CEMIN, J. G.; FERNANDES, M. B. Composição florística de um fragmento florestal ciliar no Sul de Santa Catarina. Tecnologia e Ambiente, v. 18, p. 55-70, 2014.

DUFLOTH, J. H.; BACK, Á. J. Déficit Hídrico em Pastagens na Região de Sombrio, SC, Tecnologia e Ambiente, v. 18, p. 44-55, 2012.

FRUCHTERMAN, T.; REINGOLD, E. Graph Drawing by Force-Directed Placement. Software: Practice and Experience, v. 21, n. 11, p. 1129-1164, 1991.

GARCIA, L. P.; DUARTE, E. Equidade de sexo e gênero na pesquisa e na publicação científica. Epidemiologia e Serviços de Saúde, v. 26, n. 3, p. 431-432, 2017.

GOULARTI FILHO, A. As origens e a diversificação do Parque Industrial Sul Catarinense. Tecnologia e Ambiente, v. 2, n. 1, p. 17-30, 1996.

GUADAGNIN, M. R.; DAL PONT, C. B.; VALVASSORI, M. L.; WESSLER, L. Inclusão de catadores em Programas de Gerenciamento Integrado de Resíduos Sólidos no Sul Catarinense: Acafor um caminho e exemplo possível. Tecnologia e Ambiente, v. 20 p. 107-131, 2014.

LADWIG, N. I.; DAGOSTIM, V.; BACK, A. J. A Evolução da paisagem da região carbonífera do Estado de Santa Catarina realizado com imagens de satélite. Revista Ra'e Ga, v. 43, p. 93-107, 2018.

LOPÉZ, W. L. ¿Puede la cienciometría contribuir a la evaluación de la producción intelectual? Universitas Psychologica, v. 16, n. 4, p. 1-5, 2017.

LOTKA, A. The frequency distribution of scientific productivity. Journal of the Washington Academy of Sciences, v. 16, p. 317-323, 1926.

MACHADO, A. M.; FANJUL, N. N. J.; LOPÉZ, R. B.; POVEDANO, N. A. Análisis bibliométrico de la revista RELIME (1997-2011). Investigación bibliotecológica, v. 29, n. 99, p. 91-104, 2015.

MAFFIOLETTI, N. S.; ROSSATO, A. E.; DAL-BÓ, S.; AMARAL, P. A.; CITADINIZANETTE, V. Bauhinia forficata Link (Fabaceae) no combate ao diabetes mellitus: aspectos taxonômicos, agroecológicos, etnobotânicos e terapêuticos. Tecnologia e Ambiente, v. 18, p. 1-18, 2012.

MARCOMIN, F. E.; CITADINI-ZANETTE, V. Levantamento florístico preliminar da vegetação arbórea ciliar do rio tubarão, em perímetro urbano do município de Tubarão, SC. Tecnologia e Ambiente, v. 5, n. 1. p. 31-132, 1999.

MATIAS, C. P. P.; OOSTERBEEK, L. M.; CURA, P.; DOS SANTOS, J.; SANTOS, M. C. P.; CAMPOS, J. B. Andakatu: Educação Patrimonial Interativa. Tecnologia e Ambiente, v. 17, p. 26-37, 2018.

MELO, G.; MISAEL, G. Y. M.; SIQUEIRA, N. Indicadores de sustentabilidade urbana na Bacia do Rio Belém, Vila Hauer, Curitiba, PR. Tecnologia e Ambiente, v. 24, p. $197-$ 214, 2018.

MELO, H. P.; LASTRES, H. M. M.; MARQUES, T. C. D. Gênero no sistema de ciência, tecnologia e inovação no Brasil. Revista Gênero, v. 4, n. 2, p. 73-94, 2004. 
MILIOLI, G. Planejamento, avaliação da tecnologia e desenvolvimento sustentável. Tecnologia e Ambiente, v. 1, n. 2, p. 103-112, 1995.

MONGE-NÁJERA, J.; HO, Y. S. Bibliometry of the Revista de Biología Tropical/International Journal of Tropical Biology and Conservation: Document types, languages, countries, institutions, citations and article lifespan. Revista de Biología Tropical, v. 64, n. 3, p. 1223-1235, 2016.

NIMMO, E. Colonization and cultural interaction: exploring the expression and continuation of ethnic identity in spanish colonial female religious institutions. Tecnologia e Ambiente, v. 19, p. 210-226, 2013.

PAVEI, D. D.; CAMPOS, J. B.; ZOCCHE, J. J.; SANTOS, M. C. P. Zooarqueologia de vertebrados do Sambaqui do Papagaio, Bombinhas, Santa Catarina. Tecnologia e Ambiente, v. 21, p. 70-89, 2014.

OLIVEIRA, J. M. Áreas de risco geológico/geotécnico: a experiência de Porto Alegre, RS. Tecnologia e Ambiente, v. 2, n. 2, p. 7-10, 1996.

PEZENTE, D. P.; SAN'T ANA, W. O. Evolução da fertilidade de solos construídos em áreas de passivo ambiental da mineração de carvão, Lauro Muller, SC. Tecnologia e Ambiente, v. 24, p. 146-158, 2018.

QUEIROZ, G. C. O carvão catarinense no cenário energético internacional. Tecnologia e Ambiente, v. 1, n. 1, p. 13-28, 1995.

R CORE TEAM. 2018. R: A language and environment for statistical computing. R Foundation for Statistical Computing, Vienna, Austria. URL: www.R-project.org.

PADILHA, P. T.; ELIAS, G. A.; AZEREDO, T. E. V.; COLARES, R. C.; ANTUNES, A. R.; CITADINI-ZANETTE, V. Comunidade de bromélias epifíticas em um fragmento florestal urbano no Sul de Santa Catarina, Brasil. Tecnologia e Ambiente, v. 22, p. 82$100,2016$.

PATRÍCIO, R. S.; PEREIRA, A. S.; ELIAS, S. G.; FURLANETTO, C. B.; CASCAES, M. F.; VINHOLES, A. R.; ROSADO, J. L. O.; MARQUES, B. H. Apifauna (Hymenoptera: Apidae) em Área de Restinga no Sul de Santa Catarina, Brasil. Tecnologia e Ambiente, v. 20, p. 248-269, 2014

ROSADO, J. L. O.; BECKMANN, G. H.; PATRÍCIO, R. S.; MARQUES, B. H. Estrutura da Assembleia de Formigas (Hymenoptera: Formicidae) Epigéicas em áreas reabilitadas após mineração de carvão a céu aberto no Sul de Santa Catarina, Brasil. Tecnologia e Ambiente, v. 20, p. 207-227, 2014.

SANTOS, R.; KLEIN, A. S.; CITADINI-ZANETTE, V.; PEREIRA, J. L.; C. J. Composição florística de fragmento urbano de Floresta Ombrófila Densa em Morro Casagrande, Município de Criciúma, Santa Catarina. Tecnologia e Ambiente, v. 12, n. 1, p. 103-119, 2006.

SCHWENGBER, V. L. Novas Tecnologias de informação e arqueologia: contribuições para a educação patrimonial. Tecnologia e Ambiente, v. 17, p. 121-132, 2011.

SOARES, A, L.; DIAS, G. Comunidades, identidades e sua sustentabilidade econômica: valorização do patrimônio através de uma práxis educativa. Tecnologia e Ambiente, v. 17, p. 4-25, 2011. 
SILVA, G. T.; ROSSO, P. Malacofauna Macroscópica nos Costões Rochosos da Praia da Ribanceira, Imbituba, Santa Catarina. Tecnologia e Ambiental, v. 20, p. 81-92, 2014.

SPILLERE, D. W.; PEREIRA, J. L. Análise da paisagem aplicada ao planejamento territorial de unidades de conservação: estudo de caso da Área de Proteção Ambiental do Morro Albino e Esteves. Tecnologia e Ambiente, v. 23, p. 122-131, 2017.

Tecnologia e Ambiente (2019). Sobre a tecnologia e Ambiente. Disponível em: http://periodicos.unesc.net/tecnoambiente. Acesso em: 18 mar. 2018.

VINHOLES, A. R. ; DELFINO, L. S.; BIANCO, A.; HERTER-MARQUES, B.. Fenologia da frutificação e estratégias de dispersão de árvores e arbustos em um fragmento urbano no Sul de Santa Catarina, Brasil. Tecnologia e Ambiente, v. 21, p. 223-242, 2015.

VIRTUOSO, J. C.; MILIOLI, G. Tecnologia e Sociedade: impactos socioambientais da telefonia. Tecnologia e Ambiente, v. 12, p. 39-72, 2006.

ZHENG, X.; LIU, Y. J.; HU, W. H.; HUANG, H.; NI, Y. P.; ZHAO, H. N.; JIN, Z. Z.; ZHANG, C. C.. Bibliometrics study on the Journal of American. College Health, v. 4, n. 3, p. 133-140, 2017.

WITT, A.; BORGES, J. C.; CORRÊA, J. M.. Arroio da Pescaria como Patrimônio Histórico Urbano no Município de Capão da Canoa, RS. Tecnologia e Ambiente, v. 24, p. 95-115, 2018. 